\title{
Outcomes of Clostridioides difficile Infection in Patients With Liver Cirrhosis: A Nationwide Study
}

\author{
Abubaker O. Abdalla ${ }^{\mathrm{a}, \mathrm{c}}$, Sailaja Pisipati ${ }^{\mathrm{b}}$, Mohamed Elnaggar ${ }^{\mathrm{b}}$, Mohit Rishi ${ }^{\mathrm{b}}$, \\ Rajkumar Doshi ${ }^{b}$, Nageshwara Gullapallib
}

\begin{abstract}
Background: Clostridioides difficile infection (CDI) is a major health problem that has been on the rise for the last couple of decades. It has significant mortality and morbidity in hospitalized patients. We looked at the outcomes of CDI in patients with liver cirrhosis compared to those without liver cirrhosis.
\end{abstract}

Methods: We conducted a retrospective study from a large inpatient database. The National Inpatient Sample (NIS) was queried for CDI admissions between January 2012 and September 2015. Patients admitted with CDI were identified using the International Classification of Diseases, Ninth Revision, Clinical Modification codes. The outcomes included overall mortality, length of hospitalization, and healthcare expenditure related to hospitalization.

Results: Out of all patients, 53,765 (3.97\%) had concurrent CDI and liver cirrhosis. Diabetes mellitus, alcohol abuse, and acquired immunodeficiency were observed more in patients with liver cirrhosis. Overall mortality (adjusted odds ratio (aOR) 1.65, 95\% confidence interval (CI) $1.53-1.77, \mathrm{P}<0.001$ ), cost of hospitalization and length of hospital stay (11.0 vs. 10.4 days, $\mathrm{P}<0.001)$ were significantly higher in patients with cirrhosis.

Conclusions: Patients with CDI and liver cirrhosis have significantly higher mortality, prolonged hospitalization and healthcare expenditure. Further studies are recommended to look at reversible risk factors for CDI in patients with liver cirrhosis to guide quality measures that would ultimately improve outcomes.

Keywords: Clostridioides difficile; Cirrhosis; Hospital outcomes; Mortality

Manuscript submitted November 17, 2019, accepted February 8, 2020

aDepartment of Hospital Internal Medicine, Mayo Clinic, Rochester, MN 55905, USA

bDepartment of Internal Medicine, University of Nevada, School of Medicine, 1155 Mill St W11, Reno, NV 89502, USA

${ }^{\mathrm{c} C o r r e s p o n d i n g ~ A u t h o r: ~ A b u b a k e r ~ O . ~ A b d a l l a, ~ D e p a r t m e n t ~ o f ~ H o s p i t a l ~ I n t e r-~}$ nal Medicine, Mayo Clinic, 200 First Street SW, Rochester, MN 55905, USA. Email: Abubaker.omer@gmail.com

doi: https://doi.org/10.14740/gr1240

\section{Introduction}

Clostridioides difficile is a gram-positive bacterium that was initially thought to be benign and isolated from the fecal flora of healthy newborns [1]. Bartlet [2] described its association with pseudomembranous colitis, and by the late 1980s, his team described multiple aspects of this illness. In the USA, Clostridioides difficile infection (CDI) is a common healthcare-associated infection (HCAI). The incidence of CDI has increased dramatically over the past two decades [3]. Multiple CDI outbreaks in acute care facilities have been attributed to the North American pulsed-field gel electrophoresis type 1 (NAP1) strain $[4,5]$. Broad-spectrum antibiotics, prolonged hospitalization, advanced age, renal insufficiency, multiple comorbidities, immunosuppression, use of proton pump inhibitors (PPIs), and hypoalbuminemia are some of the widely recognized risk factors for CDI [4, 6-8].

Patients with liver cirrhosis have poor immunity, weaker immune response during illness, and a propensity for increased bacterial translocation and endotoxemia [9]. CDI is an independent mortality risk factor in patients with chronic liver disease similar to hepatic encephalopathy, spontaneous bacterial peritonitis and variceal bleeding [10]. Patients with liver cirrhosis and concurrent CDI have worse outcomes when compared to those without CDI. We conducted this study to assess the outcomes of CDI in patients with and without liver cirrhosis between 2012 and 2015.

\section{Materials and Methods}

\section{Data source}

The National Inpatient Sample (NIS) database is one of the largest inpatient databases that includes more than 4,000 hospitals and an estimated average of eight million admissions annually. It provides a large number of clinical and nonclinical variables from many hospitals that can be utilized to estimate outcomes for various clinical conditions and patient populations [11].

\section{Study design}

We used the International Classification of Disease, Ninth Re- 


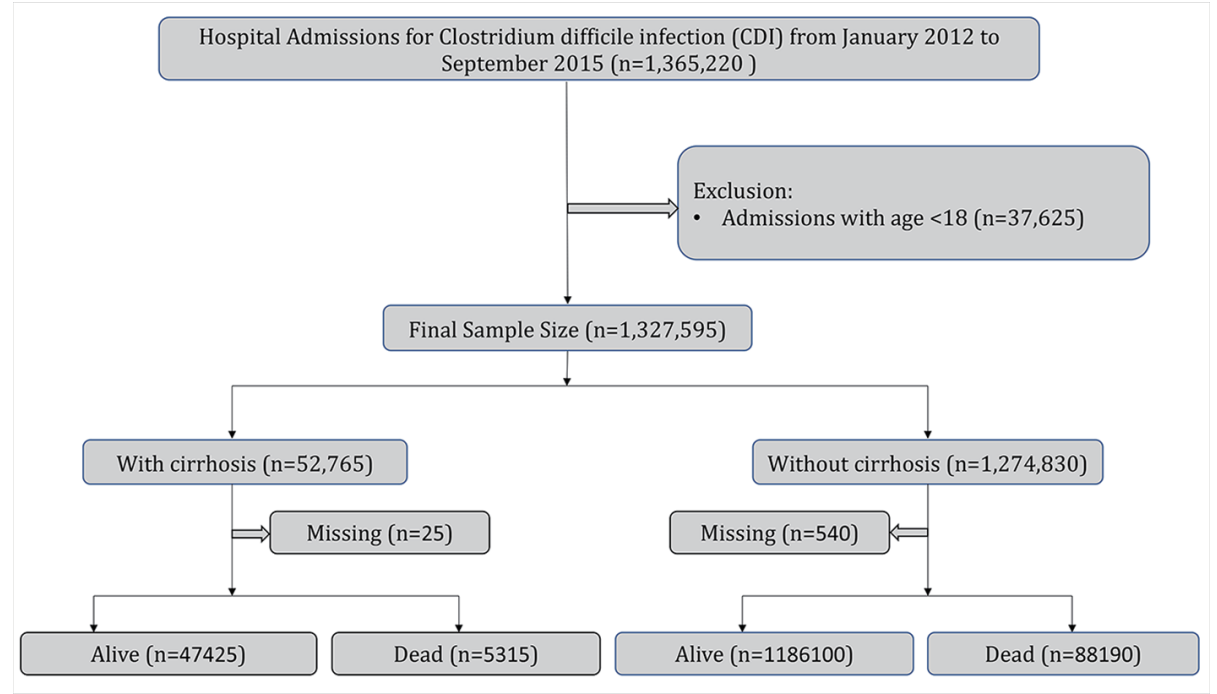

Figure 1. Population derivation.

vision, Clinical Modification (ICD-9-CM) to select our study population. Patients admitted with CDI as a primary or secondary diagnosis were identified using ICD-9-CM code 008.45. Only patients who were admitted between January 2012 and September 2015 were included in the study as the administrative data for hospitals started implementing ICD-10-CM in October 2015. Patients with a history of liver cirrhosis were identified using the ICD-9-CM codes 571.2, 571.6 and 571.5 (Fig. 1). We looked at the in-hospital mortality, length of stay (LOS), and hospitalization as the final outcomes. We also collected demographic information in addition to other comorbid conditions and risk factors.

\section{Statistical analysis}

NIS database was analyzed using SAS 9.4 (SAS Institute Inc., Cary, NC, USA). Continuous variables were described in the form of means \pm standard deviations (SDs). Chi-square test and Student's $t$-test were utilized to analyze categorical and continuous variables, respectively. Adjusted in-hospital mortality was calculated by multivariate logistic regression analysis. Patient variables, hospital characteristics, and comorbid conditions were analyzed using multivariate models. Statistical significance was set at a two-sided value of $\mathrm{P}<$ 0.05 .

\section{Results}

A total of $1,327,595$ patients with CDI were hospitalized between January 2012 and September 2015. Of them, 3.97\% $(52,765)$ had a history of concurrent liver cirrhosis. The mean age for patients with liver cirrhosis was 60 years compared to 67 years for those without a history of liver cirrhosis. Most of our patients were Caucasians (71\%) and women (58\%). Diabetes mellitus, alcohol abuse, acquired immunodeficiency, and solid tumors were noted significantly more in patients with liver cirrhosis. Patients with cirrhosis were more likely to be admitted to large urban teaching hospitals $(66.1 \%$ vs. $58.2 \%, \mathrm{P}$ $<0.001)$ and to have private insurance $(19.5 \%$ vs. $17.6 \%, \mathrm{P}<$ $0.001)$. The inpatient mortality was higher in patients with CDI and cirrhosis $(10.1 \%$ vs. $6.9 \%, \mathrm{P}<0.001)$. The LOS (11 days vs. 10.4 days $)$ and cost $(23,055$ vs. 26,535$)$ were also significantly different between the two groups after adjusted analysis (Tables 1, 2).

\section{Discussion}

Almost 4\% of all admitted patients with CDI had a history of liver cirrhosis. This is lower than the prevalence of liver cirrhosis in the general population (4.5\% to 9.0\%) [12]. The in-hospital mortality in patients was significantly higher in patients with CDI and liver cirrhosis when compared to the cohort without cirrhosis (odds ratio (OR) 1.65, 95\% confidence interval (CI) $1.53-1.77, \mathrm{P}<0.001)$ after adjusting for patient-level variables, comorbidities and hospital-level characteristics. We adjusted for alcohol abuse, immunodeficiency, toxic megacolon, metastatic cancer, congestive heart failure and chronic renal disease. Bajaj et al [10] demonstrated that mortality in patients with liver cirrhosis and CDI (13.8\%) was significantly higher than in patients with cirrhosis $(8.2 \%)$ or CDI $(9.6 \%)$ alone. Rosenblatt et al [13] found CDI to be an independent risk factor for mortality in the same patient population. This could possibly be related to generalized suppression of defense mechanism associated with liver failure, effects of hypoalbuminemia and increased risk of bacterial translocation resulting in spontaneous bacterial peritonitis in patients with chronic liver disease.

Smith et al [14] reported prolonged LOS in patients with liver cirrhosis and CDI when compared to patients without CDI (15.1 days vs. 6.55 days). Bajaj et al [10] noted that LOS was as twice as long in patients with cirrhosis and CDI, compared 
Table 1. Baseline Characteristics of Index Hospitalizations Secondary to CDI

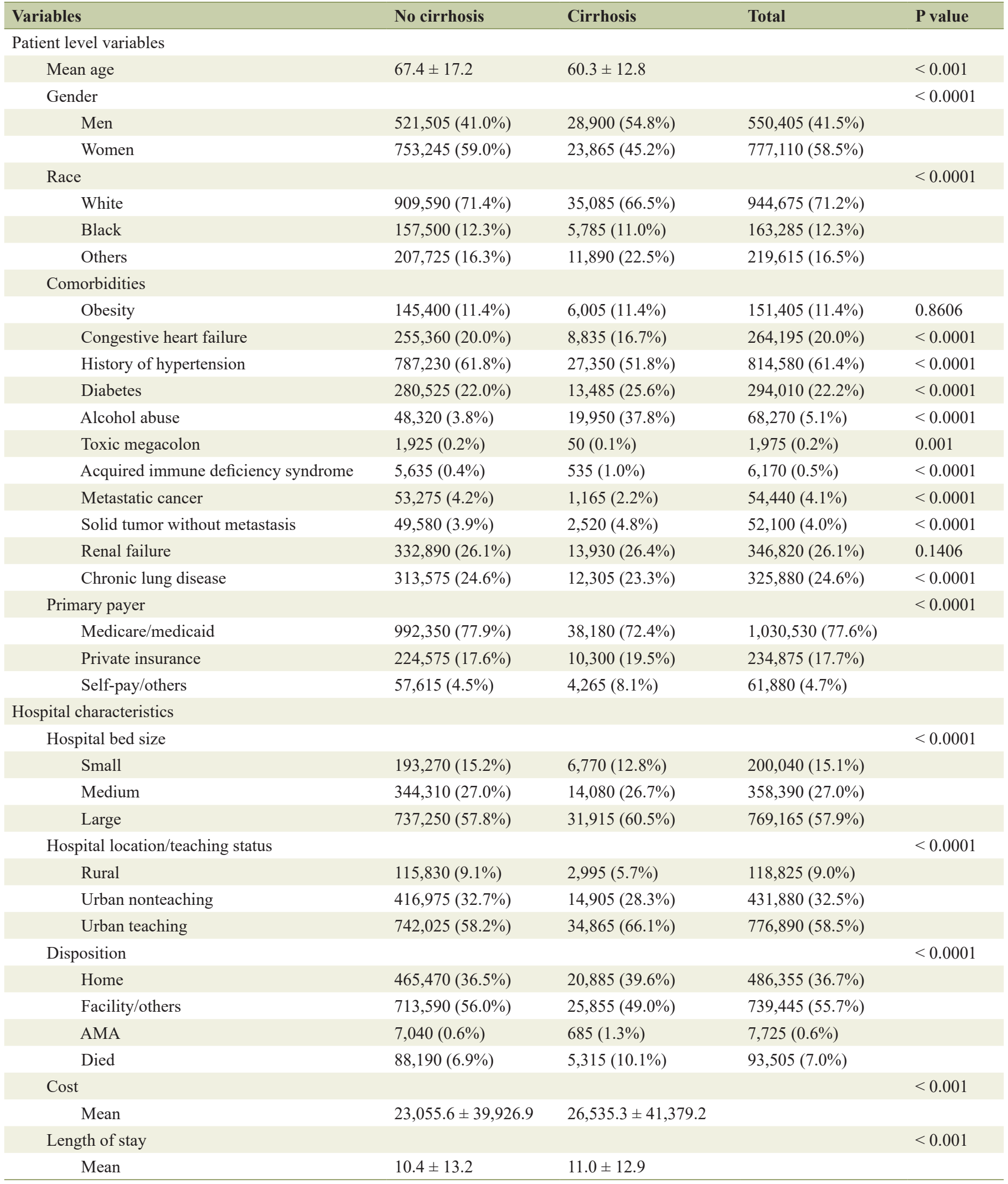

AMA: against medical advice; CDI: Clostridioides difficile infection. 
Table 2. Adjusted Mortality of CDI Admissions

\begin{tabular}{lllll}
\hline Outcomes (mortality) & Adjusted odds ratio & Lower limit & Upper limit & P value \\
\hline Without cirrhosis & Reference & Reference & Reference & 1.77 \\
With cirrhosis & 1.65 & 1.53 & $<0.001$ & \\
\hline
\end{tabular}

Adjusted with age, gender, hospital level characteristics (hospital type, bed-size) and comorbidities (obesity, congestive heart failure, hypertension, diabetes mellitus, metastatic cancer, immunodeficiency, alcohol abuse, toxic megacolon, renal failure, chronic lung disease). CDI: Clostridioides difficile infection.

to patients without CDI (14.4 days vs. 6.7 days). The length of hospital stay was prolonged for patients with CDI and liver cirrhosis as compared to those without cirrhosis but only by a minimal margin (11 days vs. 10.4 days). Although the difference was statistically significant, the effect of cirrhosis on the LOS for patients with CDI does not seem that remarkable as the effect of CDI on patients with cirrhosis. The reasons for the discrepancy in the observed results remain unclear and limited by the nature of the NIS database.

The cost associated with in-hospital stay was significantly higher in patients with cirrhosis than in those without among the entire cohort with CDI. Dotson et al [15] conducted a similar study on the same database from 2009 and found similar findings. This could be related to complications secondary to chronic liver disease that resulted in further diagnostic and therapeutic endoscopies, admission to the intensive care unit (ICU) and additional treatments. It is hard to tell how much of the cost could be attributed directly to the LOS since the difference between the two groups was not that remarkable when it came to LOS. Overall, the outcomes of CDI in patients with liver cirrhosis were worse when compared to patients without liver cirrhosis. The impact of liver cirrhosis on LOS in patients with CDI is not as notable as that of CDI in patients with liver cirrhosis.

\section{Limitations}

The study is limited by utilizing the discharge diagnoses and the billing codes of the NIS database that subject the data to inaccuracy related to under documentation and missing codes. There were significant differences in patient-level variables, hospital characteristics and comorbidities between the two groups at baseline. We aimed to limit the effect of difference at baseline by running a multivariate analysis, but this would not account for all possible confounding factors between the two groups. We could not assess the CDI severity, stage of liver cirrhosis, use of prophylactic antibiotic therapy, invasive and surgical interventions of our study sample.

\section{Conclusions}

To our knowledge, this is the largest study to date looking at the outcomes of CDI in patients with liver cirrhosis. Patients with CDI and liver cirrhosis have significantly higher mortality, prolonged hospitalization and healthcare expenditure. Further studies are recommended to look at reversible risk factors for CDI in patients with liver cirrhosis to guide quality meas- ures that would ultimately improve outcomes.

\section{Acknowledgments}

None to declare.

\section{Financial Disclosure}

None to declare.

\section{Conflict of Interest}

None to declare.

\section{Informed Consent}

Not applicable.

\section{Author Contributions}

AOA contributed to manuscript writing and revision; SP and MR were involved in literature review and manuscript writing; $\mathrm{RD}$ contributed to data extraction and analysis; NG contributed to manuscript revision.

\section{Data Availability}

Any inquiries regarding supporting data availability of this study should be directed to the corresponding author.

\section{References}

1. Hall IC, O'toole E. Intestinal flora in new-born infants: with a description of a new pathogenic anaerobe, bacillus difficilis. American Journal of Diseases of Children. 1935;49(2):390-402.

2. Gorbach SL. John G. Bartlett: Contributions to the discovery of Clostridium difficile antibiotic-associated diarrhea. Clin Infect Dis. 2014;59(Suppl 2):S66-70.

3. Khanna S, Pardi DS. The growing incidence and severity of Clostridium difficile infection in inpatient and 
outpatient settings. Expert Rev Gastroenterol Hepatol. 2010;4(4):409-416.

4. McDonald LC, Killgore GE, Thompson A, Owens RC, Jr., Kazakova SV, Sambol SP, Johnson S, et al. An epidemic, toxin gene-variant strain of Clostridium difficile. N Engl J Med. 2005;353(23):2433-2441.

5. Freeman J, Bauer MP, Baines SD, Corver J, Fawley WN, Goorhuis B, Kuijper EJ, et al. The changing epidemiology of Clostridium difficile infections. Clin Microbiol Rev. 2010;23(3):529-549.

6. Marwick CA, Yu N, Lockhart MC, McGuigan CC, Wiuff C, Davey PG, Donnan PT. Community-associated Clostridium difficile infection among older people in Tayside, Scotland, is associated with antibiotic exposure and care home residence: cohort study with nested case-control. J Antimicrob Chemother. 2013;68(12):2927-2933.

7. Loo VG, Bourgault AM, Poirier L, Lamothe F, Michaud $\mathrm{S}$, Turgeon N, Toye B, et al. Host and pathogen factors for Clostridium difficile infection and colonization. N Engl J Med. 2011;365(18):1693-1703.

8. Kwok CS, Arthur AK, Anibueze CI, Singh S, Cavallazzi $\mathrm{R}$, Loke YK. Risk of Clostridium difficile infection with acid suppressing drugs and antibiotics: meta-analysis. Am J Gastroenterol. 2012;107(7):1011-1019.

9. Sipeki N, Antal-Szalmas P, Lakatos PL, Papp M. Im- mune dysfunction in cirrhosis. World J Gastroenterol. 2014;20(10):2564-2577.

10. Bajaj JS, Ananthakrishnan AN, Hafeezullah M, Zadvornova Y, Dye A, McGinley EL, Saeian K, et al. Clostridium difficile is associated with poor outcomes in patients with cirrhosis: A national and tertiary center perspective. Am J Gastroenterol. 2010;105(1):106-113.

11. Healthcare cost and utilization project (HCUP) database overview of the national (nationwide) inpatient sample.

12. Scaglione S, Kliethermes S, Cao G, Shoham D, Durazo R, Luke A, Volk ML. The epidemiology of cirrhosis in the United States: a population-based study. J Clin Gastroenterol. 2015;49(8):690-696.

13. Rosenblatt R, Mehta A, Cohen-Mekelburg S, Shen N, Snell D, Lucero C, Jesudian A, et al. The rise of Clostridioides difficile infections and fall of associated mortality in hospitalized advanced cirrhotics. Liver Int. 2019;39(7):1263-1270.

14. Smith EZ, Northup PG, Argo CK. Predictors of Mortality in Cirrhosis Inpatients With Clostridium difficile Infection. J Clin Gastroenterol. 2018;52(8):747-751.

15. Dotson KM, Aitken SL, Sofjan AK, Shah DN, Aparasu RR, Garey KW. Outcomes associated with Clostridium difficile infection in patients with chronic liver disease. Epidemiol Infect. 2018;146(9):1101-1105. 